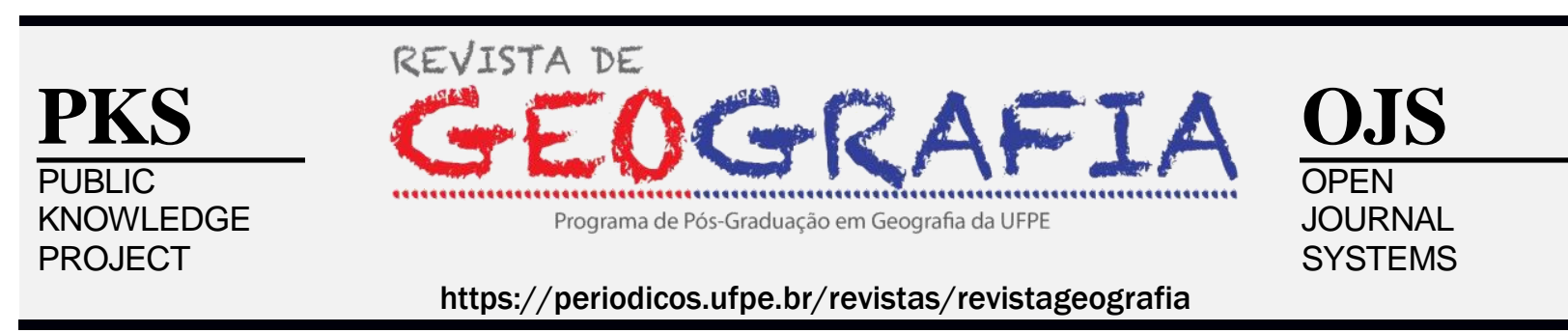

\title{
APLICAÇÃO DA ESTATÍSTICA MULTIVARIADA NA ANÁLISE DE POÇO PRODUTOR DE HIDROCARBONETO, RIO GRANDE DO NORTE/BRASIL
}

\author{
José Batista Siqueira $^{1}$
}

${ }^{1}$ Universidade Federal do Rio Grande do Norte.E-mail: oju296@gmail.com

Artigo recebido em 03/08/18 e aceito em 12/05/19

\begin{abstract}
RESUMO
A identificação de tipos litológicos ao longo de um poço é uma das importantes contribuições da geofísica aos estudos geológicos na indústria do petróleo. No entanto, não é uma tarefa trivial, visto que as medidas feitas no poço indicam as mudanças das propriedades físicas e não necessariamente as variações litológicas. Portanto este trabalho tem como objetivo fazer a associação entre as variações das propriedades físicas e os tipos litológicos no poço. Para isso foram utilizados os perfis raios gama (GR), microresistividade (MSFL), porosidade neutrão (NPHI) e sônico (Dt). Os quais são os dados provenientes da perfilagem de poços em campo produtor de hidrocarboneto, em terra na Bacia Potiguar. Para analisar os dados obtidos através da perfilagem dos poços, foram aplicadas as técnicas estatísticas multivariadas envolvendo análise de agrupamentos e componentes principais (PCA), com auxílio do software PAST 3. Os resultados obtidos permitem relacionar os dados de perfis de poço com tipos litológicos, e associá-los a intervalos de rochas selante ou reservatório nos poços. Onde são encontradas na porção superior do poço a zona de rochas selante S10 e reservatório R10, na intermediária a zona de rochas selante S14 e reservatório R14; e zona de rochas selante S30 e reservatório R30 na porção inferior. Aplicando a análise multivariada clássica à matriz de dados por zona, elas foram separadas em dois grupos, os quais no perfil de poço associam-se as rochas selante e reservatório. Na análise de componentes principais correlation biplot, as rochas também foram separadas em dois grupos. Este resultado obtido através da associação dos perfis de poços com rochas auxilia nas atividades de gerenciamento dos reservatórios nos campos produtores de hidrocarboneto, visto que serve como orientação na tomada de decisão para completar os intervalos potencialmente produtores, no espaço geográfico do campo.
\end{abstract}

Palavras-chave: Estatística multivariada, análise de agrupamentos, poço, análise de componentes principais.

\section{APPLICATION OF MULTIVARIATE STATISTICS IN THE ANALYSIS OF HYDROCARBON PRODUCER WELL, RIO GRANDE DO NORTE/BRAZIL}

\begin{abstract}
The identification of lithologic types along a well is one of the important contributions of geophysics to geological studies in the petroleum industry. However, it is not a trivial task, since the measurements made in the well indicate the changes of the physical properties and not necessarily the lithological variations. Therefore, this work has as objective to make the association between the variations of the physical properties and the lithological types in the well. For this, the gamma rays (GR), microresistivity (MSFL), neutron porosity (NPHI) and sonic (Dt) profiles were used. These are the data obtained from wellbore profiling in the hydrocarbonproducing field on the Potiguar Basin. In order to analyze the data obtained through well profiling, the multivariate statistical techniques involving cluster analysis and main components (PCA) were applied with the help of the PAST 3 software. The results obtained allow to relate the well profile data with lithological types, and associate them at intervals of sealant or reservoir rocks in the wells. Where the upper portion of the well is the
\end{abstract}


sealant rock zone S10 and reservoir R10, in the middle the sealant rock zone S14 and reservoir R14; and sealant rock zone S30 and reservoir R30 in the lower portion. Applying the classical multivariate analysis to the data matrix by zone, they were separated into two groups, which in the well profile are associated with the sealing and reservoir rocks. In the principal components analysis biplot correlation, the rocks were also separated into two groups. This result obtained through the association of well profiles with rocks is important, since it assists in the management activities of the reservoirs in the hydrocarbon producing fields, since it serves as an orientation in the decision making to complete the potentially producing intervals in the geographic space of the field.

Keywords: Multivariate statistics, cluster analysis, well, principal component analysis.

\section{INTRODUÇÃO}

Vários estudos mostram à aplicação das técnicas de análise multivariada no tratamento e interpretação de dados geofísicos de poços. Uma das aplicações mais freqüentes refere-se à identificação e separação de eletrofácies, e sua associação aos tipos litológicos interceptados no poço. Os estudos de gerenciamento de reservatórios também têm feito uso da estatística multivariada (FLEXA et al., 2004). Neste trabalho aplicam-se a análise de agrupamentos e componentes principais, com auxílio do software PAST 3 (HAMMER, 2017), aos dados de perfis geofísicos de poços produtores de hidrocarboneto em campo de terra da bacia Potiguar/RN (Figura 01).

A exemplo de outras bacias das margens equatorial e leste brasileiras, a bacia Potiguar teve sua origem relacionada à ruptura do supercontinente Gondwana. Nessa bacia as rochas geradoras e reservatórios estão relacionados à tectônica das fases rift e drift, que foram responsáveis pela sua evolução (MATOS, 1992).

Campos petrolíferos alojam-se ao longo do trend de Carnaubais, controlados pelos efeitos da evolução desse segmento geotectônico, a qual culminou com a implantação da bacia Potiguar (Figura 01). A estrutura do campo analisado neste trabalho, que controla a acumulação, configura um anticlinal assimétrico, definido pela interação entre as camadas que mergulham para norte e a Falha de Carnaubais ao sul. Essa estrutura em parte desenvolveu-se a partir de feições herdadas do embasamento (SIQUEIRA, 2005; 2014), que se projetam para dentro do preenchimento sedimentar representado pelas Formações Pendência, Alagamar, Açu, Jandaira e Barreiras.

A seqüência de rochas reservatório é composta por arenitos de origem fluvial, e as rochas selantes são folhelhos de idade Cenomaniana, pertencentes à Unidade 3 da Formação Açu (CONCEIÇÃO et al., 1984; NOLLA, 1992; NOLLA et al., 1993). As rochas geradoras estão relacionadas à Formação Alagamar e as acumulações de hidrocarboneto estão alojadas nos reservatórios silisiclásticos da Formação Açu. 
Figura 01. Localização do campo produtor, área do retângulo, na Bacia Potiguar.

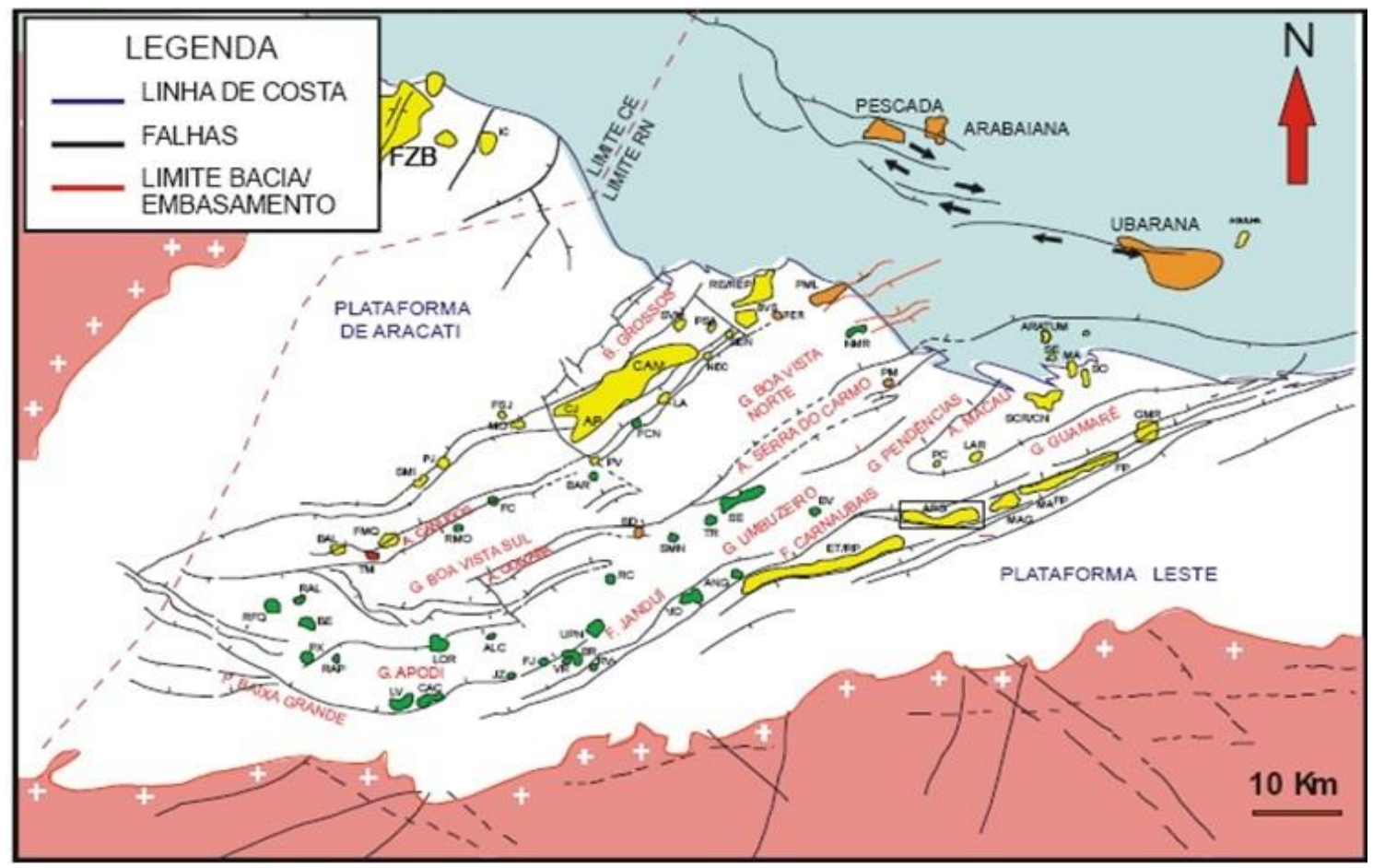

Fonte: GUSHIKEN \& SIQUEIRA, 2008 (adaptado).

A Formação Açu que aloja as principais acumulações de hidrocarboneto foi subdividida por Vasconcelos et al. (1990) em quatro unidades. As quais foram denominadas Unidade Açu 1, 2, 3 e 4. Na primeira os sistemas deposicionais predominantes foram leques aluviais gradando lateralmente para ambiente fluvial. A Unidade Açu 2 inicia com depósitos fluviais entrelaçados, gradando acima para um sistema fluvial meandrante fino. Na parte submersa da bacia, intercala-se com calcários da Formação Ponta do Mel. A base da Unidade Açu 3 é marcada por um aumento na energia deposicional, instalando-se um sistema fluvial meandrante grosso a entrelaçado; este sistema também grada acima para meandrante fino. Um afogamento regional, representado pelo Marco I, é à base da Unidade Açu 4. Esta unidade possui caráter transicional e inclui depósitos marinhos rasos. Os principais reservatórios da Formação Açu são arenitos de granulometria média a grosseira também muito grossa, excepcionalmente, os arenitos finos podem constituir-se em reservatórios produtores de hidrocarboneto. As heterogeneidades mais importantes correspondem aos níveis argilosos ou carbonáticos que ocorrem intercalados aos reservatórios. 


\section{MATERIAL E MÉTODOS}

Existem várias técnicas de estatística multivariada que podem ser aplicadas na análise de dados em diversas áreas do conhecimento, como geoquímica, geofísica, geografia dentre outras.

A análise de agrupamentos de acordo com Landim (2011) é um termo usado para descrever diversas técnicas numéricas, com o propósito de classificar valores da matriz de dados em grupos discretos. Esta técnica classificatória multivariada é utilizada quando se exploram as similaridades entre indivíduos (modo Q) ou entre variáveis (modo R), definindo-os em grupos, considerando no primeiro caso, todas variáveis medidas em cada indivíduo e no segundo dos indivíduos submetidos às mesmas mensurações.

A técnica da análise de componentes principais é o mais antigo método de ordenação, o mais conhecido e com mais exemplos de aplicação em Geociências. Trata-se, portanto de uma técnica para encontrar componentes lineares de variáveis correlacionadas por meio do cálculo de autovalores e correspondentes autovetores de uma matriz de variâncias-covariâncias ou de uma matriz de coeficientes de correlação entre variáveis.

Em vez da covariância, o coeficiente de correlação é mais usado para a matriz inicial de similaridades, porque elimina o feito de escala, pois uma variável que oscile entre 0 e 1 não pesa mais que uma que oscila entre 0 e 1000. A covariância é utilizada em variáveis com escalas idênticas ou quando se quer ressaltar as variâncias das variáveis que influenciam nas cargas fatoriais.

A análise de componentes principais não é sinônimo de análise fatorial, pois a primeira análise corresponde à transformação linear de " $n$ " variáveis originais normalmente correlacionadas entre si, em " $n$ " novas variáveis não correlacionadas, as quais são denominadas componentes principais, de modo que a primeira variável computada justifique a maior variação possível do conjunto de dados; a segunda pela maior variação restante, e assim até que toda variação do conjunto de dados seja explicada. Portanto a análise de componentes principais é uma técnica de transformação de variáveis. O método apresenta melhores resultados se a princípio já existir alguma correlação entre as variáveis, ou grupos de variáveis.

Neste trabalho buscou-se fazer a associação das variações de propriedades físicas dos tipos litológicos em profundidade utilizando como dados de entrada às informações das curvas dos perfis geofísicos de poço, obtidas a partir das lituras a cada 20,0 cm na operação de 
perfilagem. Nestes perfis estão contidas às informações referentes às variáveis raios gama (GR), microresistividade (MSFL), porosidade neutrão (NPHI) e tempo de trânsito (Dt).

A partir destes perfis sensíveis aos tipos litológicos, foi construída a base de dados, onde estas informações contidas nos perfis de poços foram compiladas em planilha Excel, e a partir disso transformadas em arquivos compatíveis com o software PAST 3 (HAMMER, 2017). No qual se selecionou conjuntos de variáveis discriminantes representativas dos padrões que diferenciam os tipos litológicos que representam rochas selantes (folhelho) e rochas reservatório (arenitos). Para analisar os dados obtidos foram aplicadas as técnicas estatísticas multivariadas envolvendo análise de agrupamentos e componentes principais (PCA) com auxílio do software PAST 3.

\section{RESULTADOS}

Análise de agrupamentos e componentes principais para as rochas selante e reservatórios por zona

Os dados do perfil de poço foram analisados seguindo sua distribuição vertical na coluna estratigráfica, da parte superior para a inferior do poço, onde são encontradas três zonas de rochas selantes e reservatórios, aqui denominadas da parte superior para a inferior do poço de zona de rochas selante S10 e reservatório R10, zona de rochas selante S14 e reservatório R14, zona de rochas selante S30 e reservatório R30. Na análise de agrupamentos os gráficos estão apresentados na horizontal, para facilitar a visualização, porém refletem a distribuição vertical dos dados obtidos com a perfilagem dos poços. Os quais serão exibidos a seguir.

\section{Zona de rochas selante $S 10$ e reservatório $R 10$}

Aplicando a análise multivariada clássica (Algoritmo UPGMA/Euclidiano, Cosntrained) à matriz de dados da zona de rochas selante S10 e reservatório R10, para as variáveis GR, MSFL, NPHI, Dt. Elas foram separadas em dois grupos, os quais no perfil de poço associamse as rochas selantes $\mathrm{S} 10$ em verde, e amarelo estão representadas as rochas reservatório R10 (Figura 02). 
Revista de Geografia (Recife) V. 36, No. 2, 2019 - ISSN: 2238-6211

Figura 02. Análise de agrupamentos UPGMA, Euclidiano, Constrained.

Zona de rochas selante S10 (cor verde) e reservatório R10 (cor amarela).

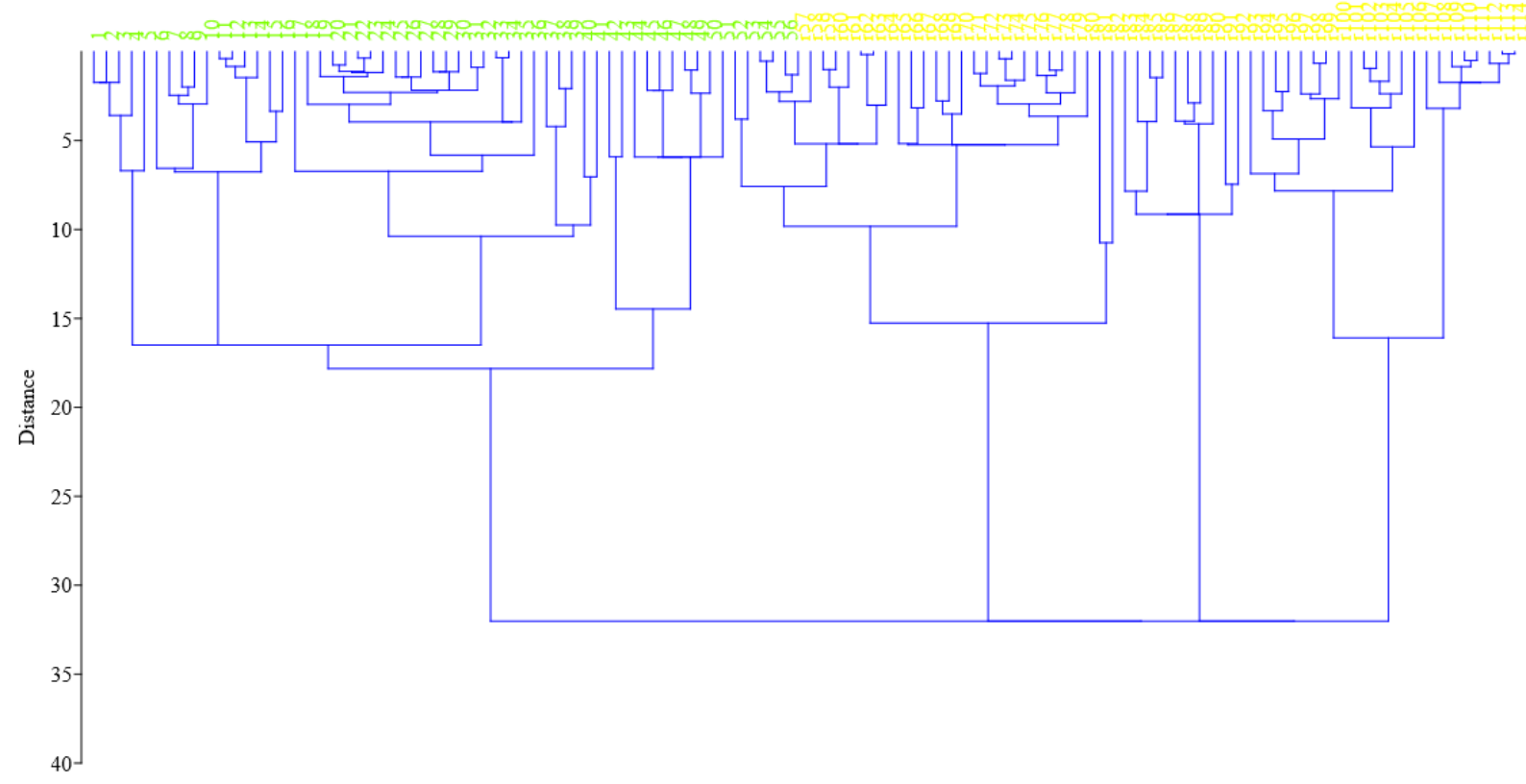

Também foi aplicada à análise de componentes principais correlation biplot, à matriz de dados, para as variáveis GR, MSFL, NPHI, Dt, elas foram separadas em dois grupos. Destacando-se em cor verde as rochas selante S10, e amarelo as rochas que no perfil de poço associam-se as rochas reservatório R10 (Figura 03). Portanto verifica-se que há boa separação entre rochas selante e reservatório nesta zona. Entretanto a sobreposição de algumas amostras das rochas reservatório com rochas selante, deve-se ao fato de que ocorrem lentes centimétricas de rochas reservatório intercaladas nas rochas selantes selante. Apesar de tratarse do marco regional mais expressivo da área (VASCONCELOS et al., 1990).

À matriz de resultados desta zona mostra que os maiores pesos para constituição do vetor 1 que corresponde a 69,57\% da variabilidade, é a variável raio gama. Para o vetor 2 que corresponde a 23,79\% da variabilidade, é a variável MSFL (Tabelas 01 e 02). Portanto raios gama (GR) e microresistividade (MSFL) são as variáveis mais importantes na classificação e separação de rochas selante S10 e reservatório R10 da zona. 
Figura 03. Análise de componentes principais correlation biplot. Zona de rochas selante S10 (cor verde) e reservatório R10 (cor amarela).

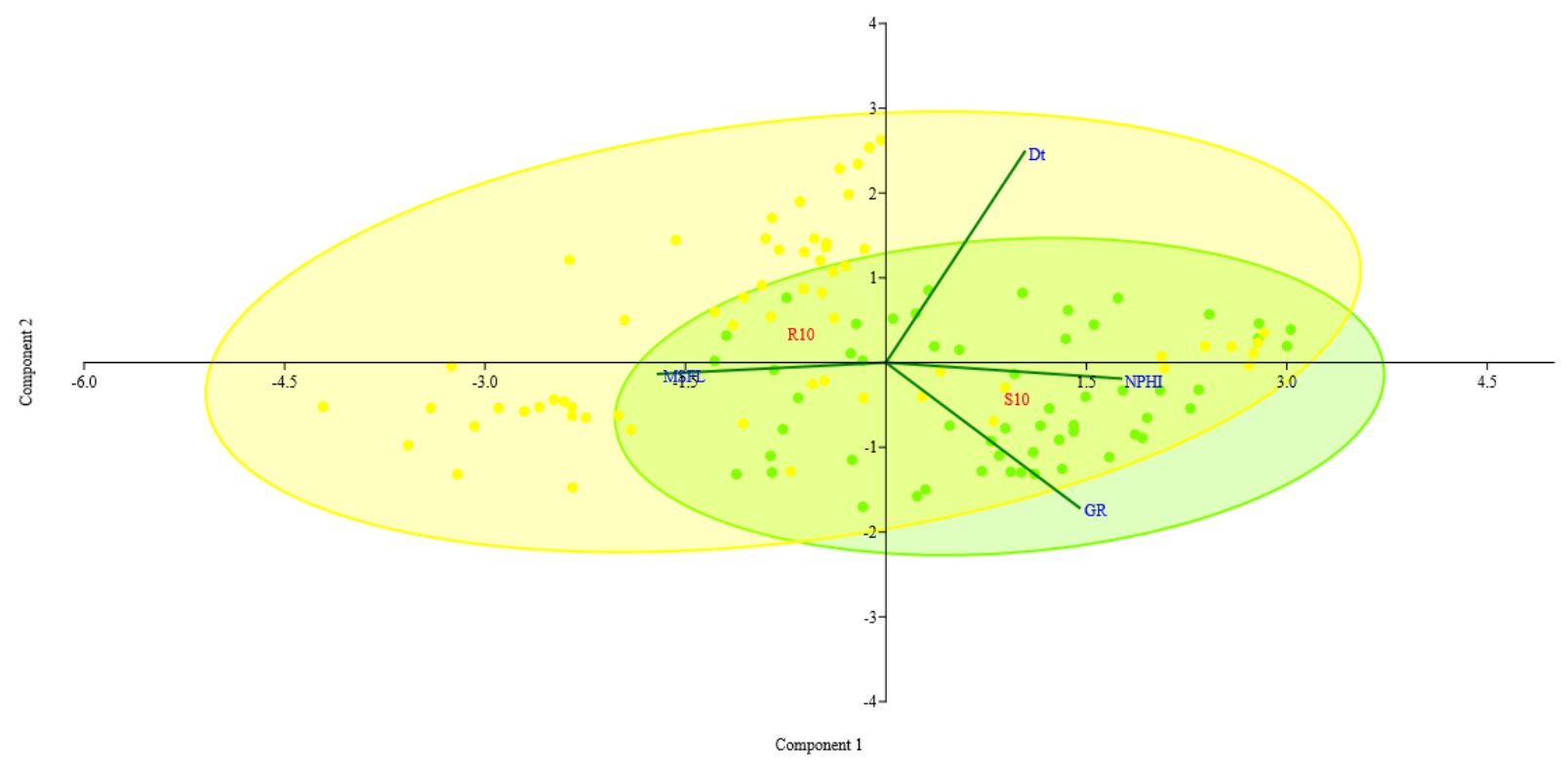

Tabela 01. Sumário da Matriz PCA (GR, MSFL, NPHI, Dt).

\begin{tabular}{crr}
\hline PC & Eigenvalue \% variance \\
1 & 2,7830 & 69,5750 \\
2 & 0,9516 & 23,7900 \\
3 & 0,1805 & 4,5121 \\
4 & 0,0849 & 2,1229 \\
\hline
\end{tabular}

Tabela 02. Pesos.

\begin{tabular}{lrrrr}
\hline Variáveis & PC 1 & PC 2 & PC 3 & PC 4 \\
GR & 0,4781 & $-0,5654$ & 0,47401 & $-0,4766$ \\
MSFL & $-0,5629$ & $-0,0445$ & 0,78146 & 0,2654 \\
NPHI & 0,5807 & $-0,0623$ & 0,14339 & 0,7990 \\
Dt & 0,3426 & 0,8213 & 0,37957 & $-0,2531$ \\
\hline
\end{tabular}

Zona de rochas selante $S 14$ e reservatório $R 14$

Aplicando a análise multivariada clássica (Algoritmo UPGMA/Euclidiano, Cosntrained) à matriz de dados da zona de rochas selantes S14 e reservatório R14, para as variáveis GR, MSFL, NPHI, Dt, elas foram separadas em dois grupos, onde na cor coral estão às rochas selantes e lilás as rochas reservatório (Figura 04). 
Zona de rochas selante $S 14$ e reservatório $R 14$

Aplicando a análise multivariada clássica (Algoritmo UPGMA/Euclidiano, Cosntrained) à matriz de dados da zona de rochas selantes S14 e reservatório R14, para as variáveis GR, MSFL, NPHI, Dt, elas foram separadas em dois grupos, onde na cor coral estão às rochas selantes e lilás as rochas reservatório (Figura 04).

Figura 04. Análise de agrupamentos UPGMA, Euclidiano, Constrained.

Zona de rochas selante S14 (cor coral) e reservatório R14 (cor lilás).

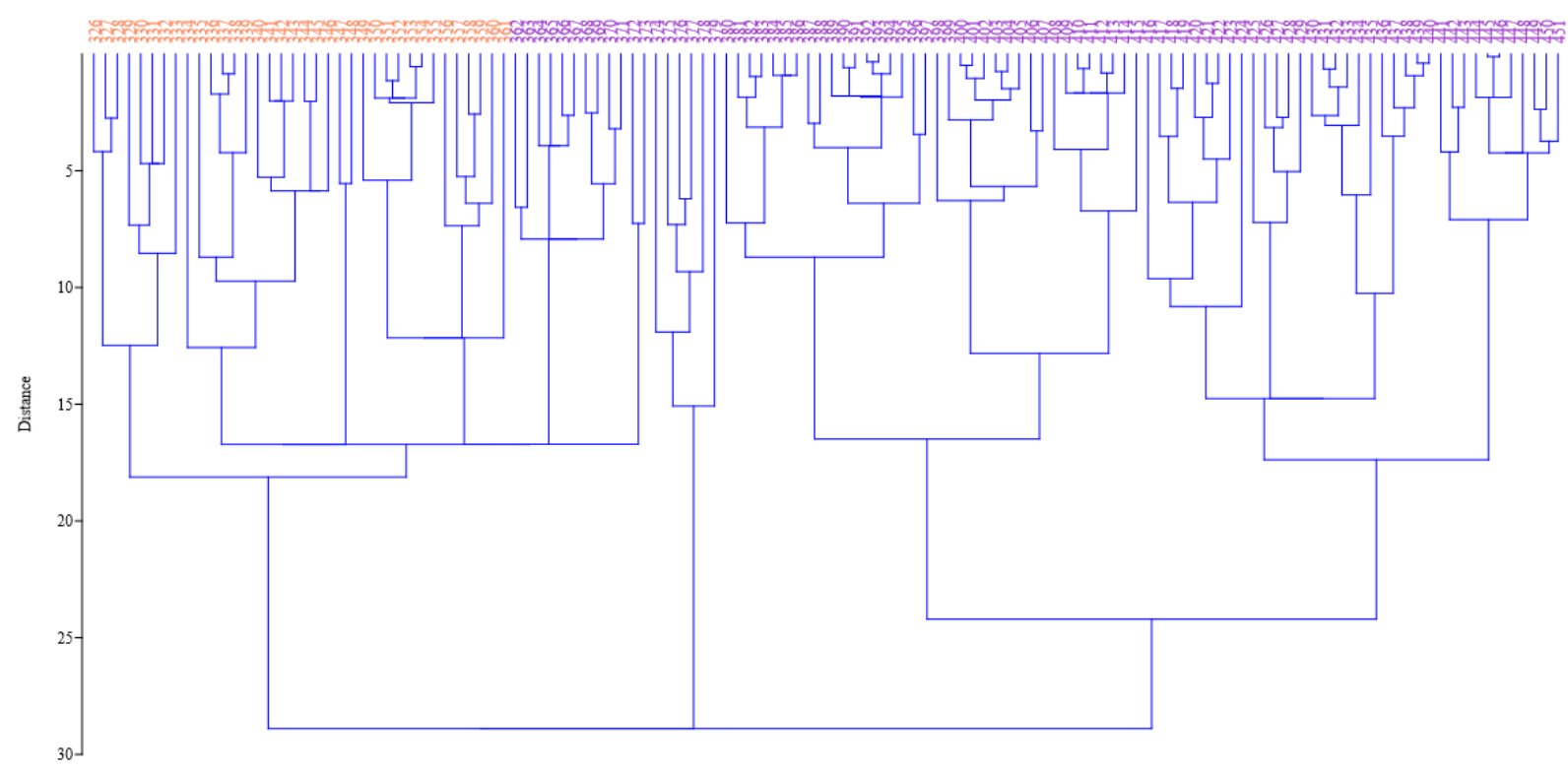

Com a aplicação da análise de componentes principais biplot à matriz de dados, as variáveis GR, MSFL, NPHI, Dt da zona selante S14 e reservatório R14, elas foram separadas em dois grupos. Os quais no perfil de poço associam-se as rochas selante S14 cor coral, e as rochas reservatório R14 lilás (Figura 05). Porém a visualização ficou melhor na análise multivariada clássica algoritmo UPGMA/Euclidiano, Constrained (Figura 04). Verifica-se certa sobreposição entre rochas selante e rochas reservatório, na análise de componentes principais dessa zona. Isto é justificada pelo fato de que há intercalações centimétricas de rochas reservatório na zona selante. E esta zona selante corresponde a um evento regional de inundação máxima de magnitude menos expressiva que a zona selante S10 (VASCONCELOS et al., 1990).

Para matriz de resultados dessa zona verifica-se que os maiores pesos para constituição do vetor 1 , que corresponde a 54,05\% da variabilidade, destaca-se a variável raios gama. Para o 
vetor 2 que corresponde a 32,39\% da variabilidade, é a variável MSFL (Tabelas 03 e 04). Portanto raios gama (GR) e microresistividade (MSFL) são as variáveis mais importantes na classificação e separação de rochas selante S14 e reservatório R14 da zona.

Figura 05. Análise de componentes principais correlation biplot. Zona de rochas selante S14 (cor coral) e reservatório R14 (cor lilás).

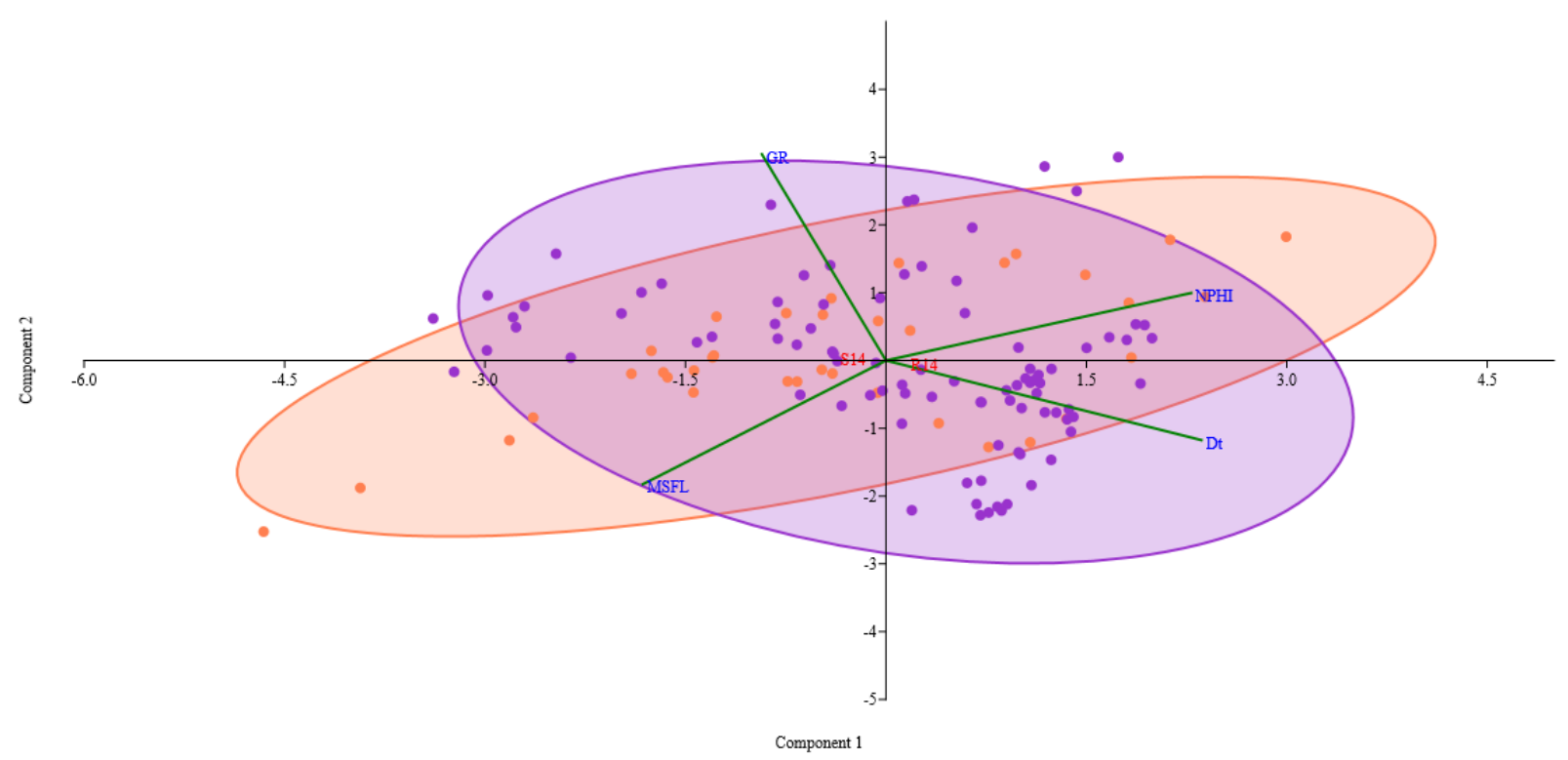

Tabela 03. Sumário da Matriz PCA das variáveis GR, MSFL, NPHI, Dt.

\begin{tabular}{crr}
\hline PC & Eigenvalue \% variance \\
1 & 2,1622 & 54,0540 \\
2 & 1,2959 & 32,3960 \\
3 & 0,4190 & 10,4750 \\
4 & 0,1230 & 30,7420 \\
\hline
\end{tabular}

Tabela 04. Pesos.

\begin{tabular}{lrrrr}
\hline Variáveis & PC 1 & PC 2 & PC 3 & PC 4 \\
GR & $-0,2400$ & 0,7867 & 0,33766 & 0,4578 \\
MSFL & $-\mathbf{0 , 4 7 0 4}$ & $-\mathbf{0 , 4 7 1 9}$ & $\mathbf{0 , 7 4 5 5 1}$ & $\mathbf{0 , 0 1 4 4}$ \\
NPHI & $\mathbf{0 , 5 9 0 5}$ & $\mathbf{0 , 2 5 7 6}$ & $\mathbf{0 , 5 4 5 9 5}$ & $-\mathbf{0 , 5 3 5 7}$ \\
Dt & $\mathbf{0 , 6 1 0 3}$ & $-0,3036$ & $\mathbf{0 , 1 7 9 2 7}$ & $\mathbf{0 , 7 0 9 4}$ \\
\hline
\end{tabular}


Zona de rochas selante $S 30$ e reservatório $R 30$

Aplicando a análise multivariada clássica (Algoritmo UPGMA/Euclidiano, Cosntrained) as amostras da zona de rochas selantes S30 e reservatório R30, elas foram separadas em dois grupos, onde em azul estão às rochas selantes S30 no perfil de poço e preto as rochas reservatório R30 (Figura 06).

Figura 06. Análise de agrupamentos algoritmo UPGMA/Euclidiano, Constrained. Zona de rochas selante S30 (cor azul) e reservatório R30 (cor preta).

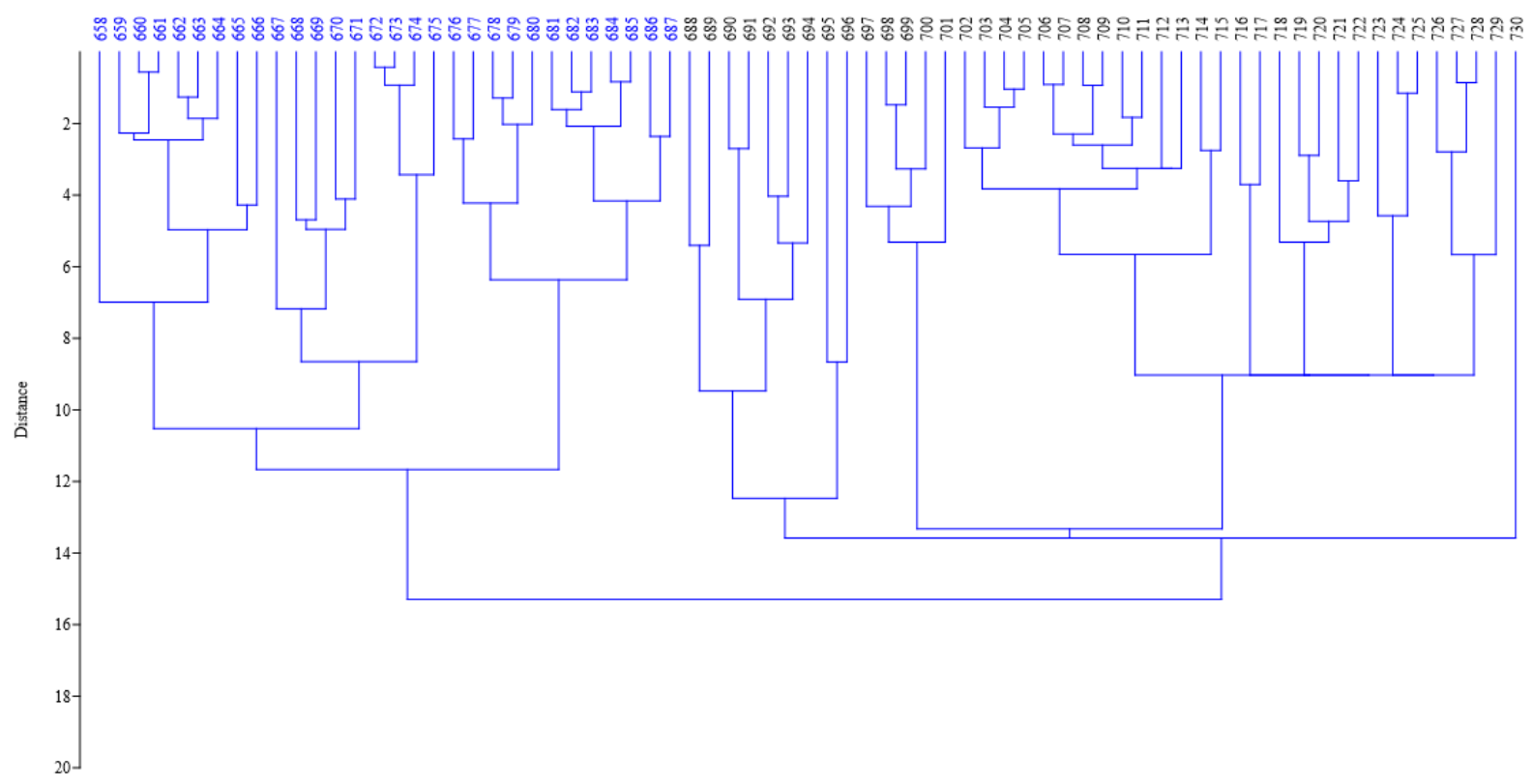

$\mathrm{Na}$ análise de componentes principais (PCA) biplot aplicada à matriz de dados, as rochas selante S30 e reservatório R30, foram separadas em dois grupos. Destacando-se em azul os tipos de rochas que no perfil de poço associam-se as rochas selante S30, e em preto as rochas reservatório (Figura 07). Entretanto alguma sobreposição é explicado pelo fato de haver intercalações centimétricas de rocha reservatório na zona selante, e uma anomalia de radioatividade nas rochas reservatório desta zona, levando-as a ser tão radioativas quanto às rochas selante constituídas essencialmente por folhelhos.

À matriz de resultados nessa zona mostra que os maiores pesos para constituição do vetor 1 que corresponde a 90,28\% da variabilidade, é a variável raio gama. Para o vetor 2 que corresponde a 9,72\% da variabilidade, é a variável MSFL (Tabelas 05 e 06). Portanto raios gama (GR) e microresistividade (MSFL) são as variáveis mais importantes na classificação e separação de rochas selante S30 e reservatório R30 da zona. 
Figura 07. Análise de componentes principais correlation biplot. Zona de rochas selante S30 (cor azul) e reservatório R30 (cor preta).

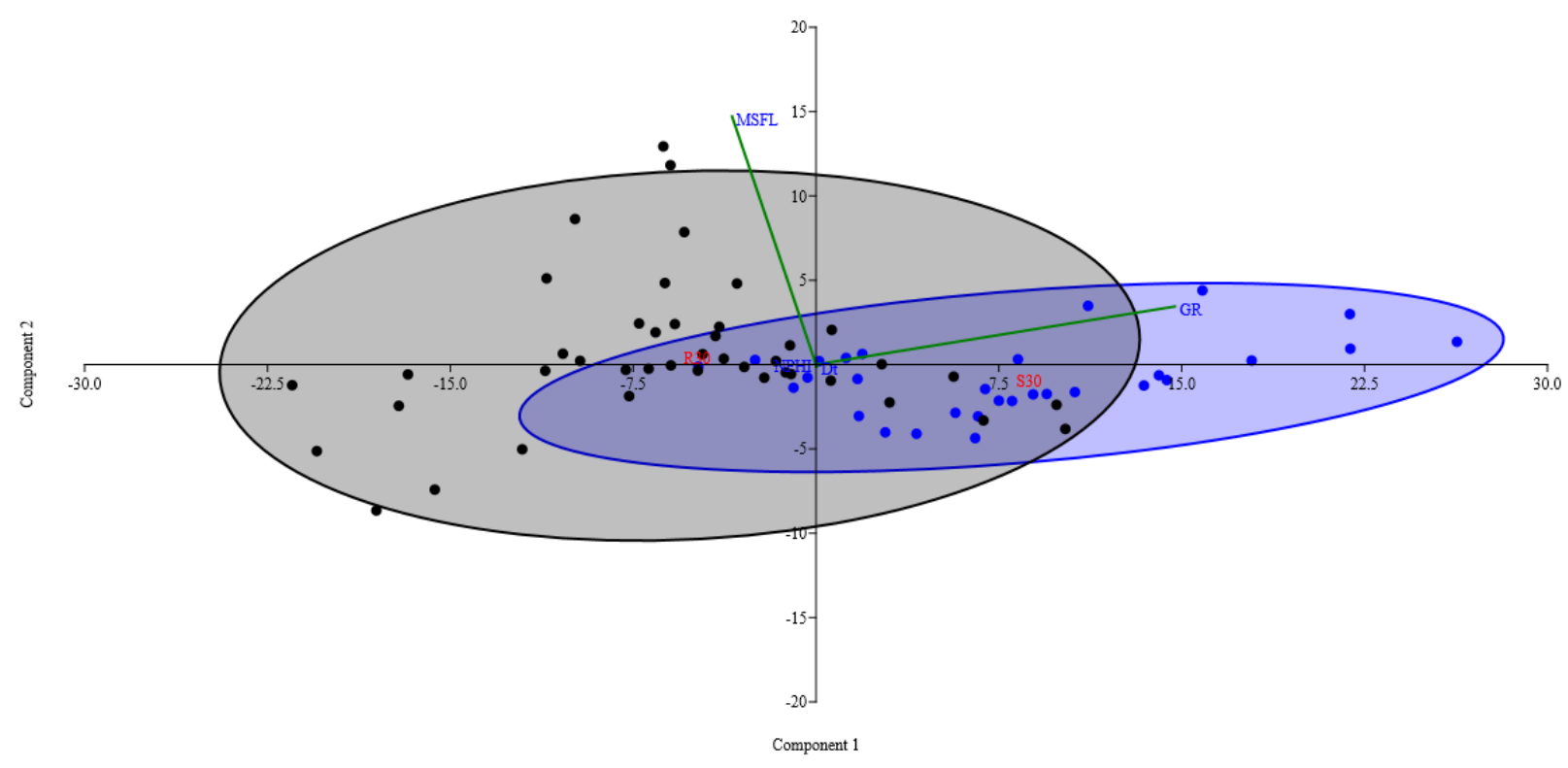

Tabela 05. Sumário da Matriz PCA das variáveis (GR, MSFL, NPHI, Dt).

\begin{tabular}{crr}
\hline PC & \multicolumn{2}{r}{ Eigenvalue \% variance } \\
1 & 100,6460 & 88,6630 \\
2 & 12,8687 & 11,3370 \\
3 & 0,0007 & 0,0006 \\
4 & 0,0004 & 0,0003 \\
\hline
\end{tabular}

Tabela 06. Pesos.

\begin{tabular}{lrrrr}
\hline & PC 1 & PC 2 & PC 3 & PC 4 \\
GR & 0,9737 & 0,2280 & 0,00113 & $-0,0007$ \\
MSFL & $-0,2280$ & 0,9736 & 0,00687 & 0,0069 \\
NPHI & 0,0023 & $-0,0074$ & 0,1333 & 0,9911 \\
Dt & 0,0002 & $-0,0060$ & 0,99105 & $-0,1333$ \\
\hline
\end{tabular}

\section{CONCLUSÃO}

Os dados de perfil de poço foram analisados seguindo sua distribuição vertical na coluna estratigráfica, da parte superior para a inferior. Onde são encontradas na porção superior do poço a zona de rochas selante S10 e reservatório R10, na intermediária a zona de rochas 
selante S14 e reservatório R14; e zona de rochas selante S30 e reservatório R30 na porção inferior.

Aplicando a análise multivariada clássica à matriz de dados da zona de rochas selante $\mathrm{S} 10$ e reservatório R10, elas foram separadas em dois grupos, os quais no perfil de poço associam-se as rochas selante e rochas reservatório. $\mathrm{Na}$ análise de componentes principais correlation biplot, as rochas também foram separadas em dois grupos. Entretanto a sobreposição de algumas amostras das rochas reservatório com rochas selante, deve-se ao fato de ocorrem lentes centimétricas de rochas reservatório intercaladas nas rochas selante. Destacando-se as variáveis raios gama (GR) e microresistividade (MSFL) como mais importantes na classificação e separação de rochas selante e reservatório na zona.

As demais zonas selante S14, reservatório R14, selante S30 e reservatório R30 apresentaram comportamentos análogos do ponto de vista de separação em grupos. Entretanto há certa sobreposição entre as rochas selante S14 e reservatório R14, na análise de componentes principais dessa zona. Justificada pelo fato de haver intercalações centimétricas de rochas reservatório na zona selante. E esta zona selante corresponder a um evento regional de inundação máxima de magnitude menos expressiva que a zona selante S10. Já na zona selante S30 e reservatório R30 alguma sobreposição entre rochas selante e reservatório, é explicada pelo fato de haver intercalações centimétricas de rocha reservatório na zona selante, e uma anomalia de radioatividade nas rochas reservatório desta zona, levando-as a ser tão radioativas quanto às rochas selante constituídas essencialmente por folhelhos.

A identificação de tipos litológicos ao longo de um poço é uma das importantes contribuições da geofísica de poço aos estudos geológicos na indústria do petróleo, conforme resultados obtidos neste trabalho, visto que isso traz informação indireta. E este conhecimento obtido através da informação dos perfis de poços é importante, pois auxilia nas atividades de gerenciamento dos reservatórios nos campos produtores de hidrocarboneto, visto que serve como orientação na tomada de decisão para completar os intervalos potencialmente produtores, no espaço geográfico do campo.

\section{AGRADECIMENTOS}

Agradecemos a Universidade Federal de Sergipe - UFS pelo apoio institucional na época, e aos revisores da revista. 


\section{REFERÊNCIAS}

CONCEIÇÃO, L.A.Z., BARROCAS, S.L., SILVA, E.J.B., GUSSO, G.L.N., SANTOS, M.A.A., SOUZA, M.S., SILVA, M.L.F., BALLIN, P.R., CAMOLEZE, Z. Projeto Alto do Rodrigues. PETROBRAS/DEBAR/CENPES. Relatório Interno. 1984.

FLEXA, R. T; ANDRADE, A.; CARRASQUILLA, A. Identificação de litotipos nos perfis de poço do Campo de Namorado (Bacia de Campos, Brasil) e do lago Maracaíbo (Venezuela) aplicando estatística multivariada. Revista Brasileira de Geociências, v.34, n.4, p.571-578. 2004.

GUSHIKEN, M.; SIQUEIRA, J. B. Características gerais dos projetos de injeção de vapor nos reservatórios produtores de petróleo da Formação Açu na Bacia Potiguar. In: XV Congresso Brasileiro de Águas Subterrâneas, Natal, Brasil. 2008. p.1-13.

HAMMER, Ø. PAleontological STatistics Version 3.15. PAST. Reference manual. Natural History Museum. University of Oslo, 253p. 2017.

LANDIM, P. B. Análise estatística de dados geológicos multivariados. São Paulo: Oficina de textos, 208p. 2011.

MATOS, R.M.D. The Northeast Brazilian Rift System. Tectonics, v.2, n. 4, p.766-791, 1992.

NOLLA, F. R. Atualização do estudo dos arenitos reservatórios da unidade 3 da Formação Açu, Campo de Alto do Rodrigues, Bacia Potiguar Emersa. PETROBRAS/DEBAR/SELAG. Nota técnica 001/92. 1992.

NOLLA, R.F.; SOUZA, C.J. de; ALVES, A.C.; FARIAS, P.R.C. de; TEIXEIRA, A.B.R.; ANJOS, S.M.; SILVA, C.F. da.; SOUZA, V.M.S.; FERNANDES, J.D.C.; BAGNOLI, E. (coord.). Petrografia e aspectos estratigráficos regionais da unidade 3 da Formação Açu na Bacia Potiguar Emersa. DEBAR/DIREX/SEALG. Minuta106-0951. 1993.

SIQUEIRA, J. B. A falha de carnaubais e o controle do Campo de Alto do Rodrigues In: X SNET IV ISIT, Curitiba, 2005. p.87-89.

SIQUEIRA, J.B. Diagnóstico do Mecanismo do influxo de águas em reservatórios siliciclásticos. In: XII Simpósio de Recursos Hídricos do Nordeste, Natal, Brasil, 2014. p.110.

VASCONCELOS, E. P.; LIMA NETO, F. F.; ROOS, S. Unidades de correlação da Formação Açu-Bacia Potiguar. In: 36 Congresso Brasileiro de Geologia, Natal, Brasil, 1990. p.227-240. 